\title{
UtilizaÇÃo de ANÁlise Multivariada e Redes NeURais ARTificiais Na Determinação do Comportamento de Colonização de POPUlaÇões de MaCRófitas AQUáticas No RESERVATÓRIO DE SANTANA ${ }^{1}$
}

\author{
Using Multivariate Statistics and Artificial Neural Networks to Determe the Colonization \\ Behavior of Aquatic Macrophyte Populations in Santana Reservoir
}

PITELli, R.L.C.M. ${ }^{2}$, FERRAUDO, A.S. ${ }^{3}$, PITELli, A.M.C.M. ${ }^{2}$, PITELLI, R.A. ${ }^{3}$ e VELINI, E.D. ${ }^{2}$

\begin{abstract}
RESUMO - As análises de agrupamento e de componentes principais e as redes neurais artificiais foram utilizadas na determinação de padrões de comportamento das populações de macrófitas aquáticas que colonizaram o reservatório de Santana, Pirai-RJ, durante o ano de 2004. As análises de agrupamento dividiram o comportamento das populações durante o ano em dois grupos distintos, apresentando um padrão no primeiro semestre que difere daquele observado no segundo semestre do ano. A análise de componentes principais demonstrou que esse comportamento da comunidade (grupo de populações) é influenciado principalmente pelas espécies S. montevidensis, Heteranthera reniformis, Ludwigia sp., Rhynchospora aurea, C. iria, C. ferax e Aeschynomene denticulata no primeiro grupo e por Echinochloa polystachya, Polygonum lapathifolium, Alternanthera phyloxeroides, Pistia stratiotes, Eichhornia azurea, Brachiaria arrecta e Oxyscarium cubense no segundo grupo. As redes neurais artificiais agruparam as populações de macrófitas aquáticas em nove grupos, conforme sua densidade nos diferentes meses do ano. A aplicação da análise de componentes principais (ACP) nos valores de frequência das populações presentes nos primeiros três grupos de Kohonen permitiu discriminar três grupos de meses, cujas populações apresentaram características diferentes de colonização. A aplicação das redes neurais artificiais permitiu melhor discriminação dos meses e das espécies que compõem as comunidades correspondentes, quando utilizada a análise de componentes principais.
\end{abstract}

Palavras-chave: análise multivariada, macrófitas aquáticas, redes neurais artificiais.

\begin{abstract}
Cluster analysis, principal components analysis and Kohonen artificial neural networks were tested to determine the behavior patterns of aquatic macrophyte colonization on Santana reservoir, Pirai-RJ, 2004. The colonization behavior of the populations was divided into two groups, representing the two year semesters. The principal components analysis showed that the behavior of the first group (first semester) was mainly influenced by $\mathbf{S}$. montevidensis, Heteranthera reniformis, Ludwigia sp., Rhynchospora aurea, C. iria, C.ferax e Aeschynomene denticulate and the second group by Echinochloa polystachya, Polygonum lapathifolium, Alternanthera phyloxeroides, Pistia stratiotes, Eichhornia azurea, Brachiaria arrecta e Oxyscarium cubense. The artificial neural networks (ANN) grouped the aquatic macrophyte populations into nine groups according to their colonization behavior during the year. The application of principal components analysis (PCA) on frequency values of the population determined by the artificial neural networks for the first three groups allowed to discriminate three groups of populations with different colonization behaviors. The application of artificial neural networks allowed a better discrimination of communities (months) and species colonization behavior than the application of PCA on raw data.
\end{abstract}

Keywords: aquatic macrophytes, multivariate analysis, artificial neural networks.

Recebido para publicação em 1.12.2008 e na forma revisada em 21.8.2009.

2 Fazenda Experimental Lageado, Faculdade de Ciências Agronômicas, UNESP, Rua José Barbosa de Barros, n. 1780, Caixa Postal 237, 18610-307 Botucatu-SP, <rlpitelli@ecosafe.agr.br>; ${ }^{3}$ Faculdade de Ciências Agrárias e Veterinárias, UNESP, Via Prof. Paulo Donato Castellane, s/n, 14884-900 Jaboticabal-SP. 


\section{INTRODUÇÃO}

Os levantamentos sistemáticos fornecem as informações necessárias para prever a evolução dos níveis de infestação e a probabilidade de ocorrência de macrófitas em condições similares às estudadas. Há várias opções para a análise estatística dos dados, incluindo o ajuste de modelos. A análise multivariada constitui-se em uma ferramenta poderosa para alcançar esses objetivos. Trata-se de um conjunto de procedimentos estatísticos que analisa múltiplas medidas de cada indivíduo ou objeto estudado; todas as variáveis devem ser aleatórias e inter-relacionadas de maneira que seus diferentes efeitos não possam ser significativamente interpretados de forma separada. As técnicas multivariadas podem ser de dependência ou interdependência (Hair et al., 2005). A técnica de dependência é aquela na qual uma variável (ou conjunto de variáveis) é identificada como variável dependente a ser predita ou explicada por outras variáveis conhecidas como independentes. Em contraste, a técnica de interdependência é aquela na qual nenhuma variável é definida como independente ou dependente; o processo envolve a análise simultânea de todas as variáveis em conjunto.

Pompêo et al. (1998) realizaram levantamentos das populações presentes na comunidade fitoplanctônica em várias profundidades no reservatório de Boa Esperança, Rio Parnaíba, na divisa entre os Estados do Maranhão e Piauí. Também foram avaliados os dados referentes a $\mathrm{pH}$, temperatura, alcalinidade, teor de oxigênio dissolvido, $\mathrm{N}$ total, clorofila e matéria seca total. O estudo estatístico, realizado por análise de agrupamentos, demonstrou que o reservatório apresenta heterogeneidade espacial horizontal com relação à diversidade de espécies de fitoplâncton. Esses dados permitiram definir dois compartimentos: a zona da barragem, com características de ambiente lêntico, elevada profundidade de penetração de luz e táxons adaptados à flutuação; e a parte alta, que apresentou características de ambiente lótico, menor transparência e maior teor de materiais de suspensão.

Colon-Gaud et al. (2004) analisaram a distribuição espacial de macroinvertebrados habitando colonizações de Hydrilla verticillata e Ceratophyllum demersum. Esses autores utilizaram a análise de componentes principais (ACP) para determinar diferenças na distribuição entre estações de crescimento e posições das macrófitas para 12 grupos taxonômicos de macroinvertebrados. A análise de componentes principais demonstrou a existência de quatro assembleias de macroinvertebrados, que explicavam $63,5 \%$ da variação nos dados. Essas assembleias incluíram Gastropoda-Hydrachnida (22,9\% da variância acumulativa), Decapoda-Odonata (13,9\%), Diptera-Coleoptera $(10,7 \%)$ e HemipteraAmphipoda (9,7\%).

As redes neurais de Kohonen ou Mapas Auto-Organizáveis (Self-Organizing Maps $S O M$ ) são modelos neurocomputacionais desenvolvidos pelo professor Teuvo Kohonen no início dos anos 80 (Kohonen, 1990). São utilizadas principalmente como análise de agrupamentos e apropriadas para trabalhar com dados envolvendo comportamentos não lineares. Pertencem à classe de modelos competitivos e não supervisionados. Em geral, esses modelos possuem inspiração neurobiológica, porém, na prática, são algoritmos computacionais, representando, de maneira bastante elementar, o mecanismo de funcionamento cerebral. O modelo é montado com base em processos de aprendizagem. Frequentemente, padrões contidos em conjuntos multivariados possuem uma estrutura complexa, podendo ser resumida preservando grande parte das informações neles contidas. Essa foi a proposta de Kohonen (Kohonen, 1989), que desenvolveu os modelos SOM facilmente interpretáveis, tornando, assim, os grupos mais facilmente caracterizáveis. A capacidade de aprender das redes neurais artificiais tem sido recentemente aplicada em ciências naturais. Em ecologia, redes neurais artificiais têm sido utilizadas para classificação de grupos (Chon et al., 1996; Levine et al., 1996), ou demonstrando relações entre variáveis (Huntingford \& Cox, 1996; Lek et al., 1996).

Costa et al. (2006) utilizaram as análises de agrupamento e de componentes principais para diferenciar as espécies Brachiaria mutica, Brachiaria subquadripara, Panicum repens, Eichhornia crassipes, Heteranthera reniformis, Typha subulata e Enhydra anagallis, utilizando dados referentes a características das 
epidermes adaxial e abaxial, feixe vascular, bainha do feixe vascular, esclerênquima, parênquima e lacunas do aerênquima, além da espessura da folha, do número de estômatos e do número de tricomas nas faces adaxial e abaxial. Os caracteres com maior poder discriminatório foram: porcentagem de epiderme adaxial (IN); porcentagem de epiderme abaxial; feixe vascular; bainha do feixe vascular; esclerênquima e lacunas do aerênquima (NC e IN); espessura da folha; e número de estômatos das faces adaxial e abaxial.

O objetivo deste trabalho foi determinar o comportamento das populações de macrófitas aquáticas que colonizavam o reservatório de Santana no ano de 2004, mediante uso das análises multivariadas de agrupamento (análise de Cluster) e de componentes principais (ACP). Objetivou-se também analisar a viabilidade de utilização das redes neurais artificiais de Kohonen na determinação de padrões comportamentais de populações de macrófitas aquáticas.

\section{MATERIAL E MÉTODOS}

\section{Levantamento das macrófitas aquáticas presentes no reservatório}

O reservatório de Santana, pertencente à Light Serviços de Eletricidade Ltda, está localizado nos municípios de Piraí, Santanésia e Barra do Piraí, no Estado do Rio de Janeiro. O mapa georreferenciado do reservatório de Santana foi cedido pelo Departamento de Meio Ambiente da empresa hidrelétrica Light. O mapa georreferenciado de reservatório foi desenhado no Datum SAD69, projeção UTM (Universal Translator Mercator), zona 23K. O mapa foi dividido em 97 áreas, de acordo com a forma da lâmina d'água. Em cada uma dessas áreas foi posicionado um ponto, que, após transferido para o GPS Garmin 76S, foi localizado no campo. Em cada um desses 97 pontos de amostragem foram avaliadas a composição específica e a densidade das populações de macrófitas aquáticas presentes no reservatório no período de janeiro a dezembro de 2004 , com exceção do mês de junho. A determinação da densidade das macrófitas aquáticas foi feita nas margens do reservatório, sendo analisada uma faixa de $10 \mathrm{~m}$ de largura (percorrendo a margem do reservatório) em cada ponto de amostragem. A caracterização da densidade das macrófitas foi realizada mediante um sistema de notas, em que: nota 0 - ausência de macrófitas no ponto; nota 1 densidade muito baixa, quando até três indivíduos de uma população foram observados no espaço avaliado de $1 \mathrm{~m}$ da região marginal; nota 2 - densidade baixa, quando a espécie foi observada em menos que $40 \%$ do segmento de $10 \mathrm{~m}$; nota 3 - densidade média, quando a espécie foi observada entre 40 e $80 \%$ do segmento; e nota 4 - densidade alta, quando a espécie foi observada em 80 a $100 \%$ do segmento.

\section{Análises multivariadas}

As técnicas multivariadas podem ser de dependência ou interdependência (Hair et al., 2005). A técnica de dependência é aquela na qual uma variável (ou conjunto de variáveis) é identificada como variável dependente a ser predita ou explicada por outras variáveis conhecidas como independentes. Em contraste, a técnica de interdependência é aquela na qual nenhuma variável é definida como independente ou dependente; o processo envolve a análise simultânea de todas as variáveis em conjunto. A abordagem utilizada neste estudo é uma técnica de interdependência, a qual será descrita mais adiante.

\section{Análise de agrupamento}

A análise de agrupamentos é uma técnica que permite classificar indivíduos em subgrupos excludentes. É um problema de otimização em que se pretende, de modo geral, maximizar a homogeneidade de indivíduos dentro de grupos e maximizar a heterogeneidade entre os grupos. A representação dos grupos é feita em um gráfico com uma estrutura de árvore, denominado dendrograma. Na análise de agrupamento usada para avaliar padrões de comportamento da comunidade no ano foi utilizado como estratégia de agrupamento o método de Ward (Ward, 1963) e, como medida de semelhança entre espécies, a distância euclidiana (coeficiente de dissimilaridade). O objetivo da análise de agrupamento é agrupar espécies ou populações que apresentam comportamentos semelhantes com base nos dados de presença e densidade das espécies. 


\section{Análise de componentes principais}

O objetivo principal da análise de componentes principais é condensar as informações contidas em um número muito grande de variáveis originais em um número menor de dimensões compostas, com uma perda mínima de informação. Esta análise realiza a redução de um número original $\mathrm{P}$ de dimensões, em que cada variável é uma dimensão, para um número menor de dimensões, em que cada dimensão é definida por uma combinação linear das $\mathrm{P}$ variáveis originais. Essas combinações lineares são denominadas componentes principais. As unidades amostrais são distribuídas em gráficos bidimensionais, onde os eixos ortogonais são os componentes principais.

\section{Redes neurais de Kohonen}

A rede de Kohonen possui duas camadas de neurônios artificiais: uma para receber os padrões de entrada e outra para gerar os padrões de saída. Os neurônios da camada de saída são dispostos em uma grade (ou mapa) retangular bidimensional, e cada um deles é conectado a todos os neurônios que compõem a camada de entrada. A similaridade entre o vetor de entradas e o vetor de pesos é expressa geralmente pela distância euclidiana. Toda vez que um padrão estimula uma vizinhança de um neurônio no mapa, o peso associado é reforçado. À medida que o processo de aprendizagem vai terminando, o raio da vizinhança vai diminuindo, armazenando nela padrões específicos, enquanto padrões externos a essa vizinhança buscam outras vizinhanças. Assim, um neurônio aprende mais sobre os padrões a ele associados, ao passo que seu vizinho aprende menos sobre esses padrões. Após o término do processo de aprendizagem, padrões de entrada similares ativarão as mesmas vizinhanças do mapa, armazenando nas vizinhanças do neurônio unidades com padrões similares. Quando uma unidade é fixada num neurônio, este é denominado neurônio vencedor (winning neuron). A vizinhança de cada neurônio pode ser definida de acordo com a forma geométrica usada para representar os neurônios da rede. O mapa algoritmo auto-organizável (SOM) - um método de redes reurais não supervisionadas - realiza as mesmas tarefas da análise de componentes principais (Kohonen, 1990). A principal utilidade desse tipo de análise é a obtenção de uma imagem objetiva das assembleias de populações com resultados influenciados pelo conhecimento do pesquisador das amostras e das características do ambiente. Os resultados de frequência das espécies fornecidos pela análise de Kohonen foram utilizados para realização de uma análise de componentes principais, para melhor visualização dos padrões de comportamento das macrófitas entre os diferentes meses do ano.

\section{RESULTADOS E DISCUSSÃO}

As espécies de macrófitas aquáticas que colonizavam o reservatório de Santana, RJ, no ano de 2004 estão apresentadas na Tabela 1, com os respectivos códigos. A Tabela 2 demonstra a soma das notas de colonização de cada espécie nos respectivos meses do ano de 2004. A análise de agrupamento utilizando matriz de dissimilaridade (distância euclidiana) construída a partir das notas de colonização das plantas nos diferentes meses do ano mostrou uma arquitetura de grupos contendo dois grupos (Figura 1): um grupo contém os meses de janeiro a maio, e o outro, de julho a dezembro.

Houve ordenação dos meses de amostragem de janeiro a dezembro. As similaridades mais intensas ocorreram nos meses de março e fevereiro, outubro e novembro, e setembro e agosto. Esse comportamento pode ser interpretado da seguinte maneira: a comunidade de macrófitas aquáticas presente em cada um dos meses mostrou características mais próximas às dos meses vizinhos, demonstrando uma variação contínua do comportamento da comunidade no tempo. A divisão observada nos dois grandes grupos pode ser explicada pela grande depleção do reservatório ocorrida em junho, com grande exposição do sedimento. Essa exposição do sedimento e consequentemente redução da profundidade em determinados pontos do reservatório propiciaram condições para o estabelecimento de algumas espécies e a redução da colonização relativa de outras. Espécies características de margens se beneficiaram com a condição, o que possibilitou maior disseminação dessas populações pelo reservatório, aumentando sua importância como um todo. 
Tabela 1 - Espécies de macrófitas aquáticas que colonizavam o reservatório de Santana no ano de 2004

\begin{tabular}{|c|c|c|c|}
\hline Espécie & Código & Espécie & Código \\
\hline Alternanthera philoxeroides & ALRPH & Pistia stratiotes & PIIST \\
\hline Brachiaria arrecta & BRASU & Polygonum lapathifolium & POLLA \\
\hline Echinochloa polystachya & ECHPO & Polygonum hydropiperoides & POLHP \\
\hline Echinochloa crus-GALLI & ECHCG & Salvinia auriculata & SAVAU \\
\hline Panicum rivulare & PANRV & Salvinia herzogii & SAVHE \\
\hline Paspalum repens & PASFL & Cyperus giganteus & CYPGI \\
\hline Hymenachne amplexicaulis & HYVAM & Cyperus iria & CYPIR \\
\hline Andropogon bicornis & ANOBI & Cyperus ferax & CYPFE \\
\hline Sagittaria montevidensis & SAGMO & Fimbristylis miliaceae & \\
\hline Egeria densa & EGEDE & Rhynchospora aurea & RHCAU \\
\hline Limnobium laevigatum & LIMST & Oxycaryum cubense & SCPCU \\
\hline Eichhornia azurea & EICAZ & Commelina diffusa & COMDI \\
\hline Eichhornia crassipes & EICCR & Thalia geniculata & THAGE \\
\hline Pontederia cordata & POFCO & Ipomoea alba & CLYAC \\
\hline Pontederia lanceolata & POFCL & Typha latifolia & THYLA \\
\hline Pontederia parviflora & & Typha domingensis & THYDO \\
\hline Heteranthera reniformis & HETRE & Hedychium coronarium & HEYCO \\
\hline Ludwigia octovalvis & LUDOC & Aeschynomene denticulata & AESDE \\
\hline Ludwigia elegans & LUDEL & Eclipta alba & ECLAL \\
\hline Ludwigia sericea & LUDSE & Enhydra anagallis & \\
\hline Myriophyllum aquaticum & MYPBR & Cabomba caroliniana & CABCA \\
\hline Potamogeton pectinatus & PTMPE & Lemna minor & LEMMI \\
\hline
\end{tabular}

Com a finalidade de discriminar as espécies que caracterizaram os diferentes grupos demonstrados pela análise de agrupamento, foi realizada uma análise de componentes principais, cujos resultados estão apresentados na Figura 2. Com 65,14\% da variância total retida nos dois primeiros componentes principais, foi possivel discriminar dois grupos de meses com comunidades que apresentam características diferentes. No primeiro grupo, localizado à esquerda do primeiro componente principal, estão os meses de janeiro, fevereiro e março e, no segundo, localizado à direita do primeiro componente principal, estão os meses de agosto, setembro, outubro, novembro e dezembro. Os meses de julho, março e abril apresentam características intermediárias entre esses dois grupos ou mesmo características de transição, o que não permitiu discriminá-los.

Na Figura 3 é apresentada a distribuição das espécies segundo os dois primeiros componentes principais. O primeiro componente principal foi o responsável pela discriminação contida na Figura 3. Assim, quanto mais próximo das extremidades do eixo $\mathrm{x}$ (primeiro componente principal) uma espécie se localizar, maior será seu poder discriminatório. Sagittaria montevidensis, Heteranthera reniformis, Ludwigia sp., Rhynchospora aurea, Cyperus iria, Cyperus ferax e Aeschynomene denticulata foram as populações que mais contribuíram para a caracterização da comunidade de plantas aquáticas nos meses de janeiro, fevereiro e março. É importante salientar que são espécies marginais que se destacaram nos levantamentos logo após a limpeza mecânica do reservatório, realizada em 2003. As populações de Echinochloa polystachya, Polygonum lapathifolium, Alternanthera phyloxeroides, Pistia stratiotes, Eichhornia azurea, Brachiaria arrecta e Oxyscarium cubense têm maior contribuição na caracterização da comunidade de macrófitas aquáticas presente nos meses de agosto a dezembro. Espécies como Eichhornia crassipes, Paspalum repens e Hymenachne amplexicaulis 
Tabela 2 - Soma das notas de colonização das macrófitas aquáticas determinadas no reservatório de Santana, Piraí-RJ, ao longo do ano de 2004. Cada valor representa a soma de 97 amostras

\begin{tabular}{|c|c|c|c|c|c|c|c|c|c|c|c|c|}
\hline Espécie & Jan. & Fev. & Mar. & Abr. & Maio & Jul. & Ago. & Set. & Out. & Nov. & Dez. & Total \\
\hline AESDE & 1 & 2 & 1 & 1 & 1 & 1 & 1 & 0 & 0 & 0 & 0 & 8 \\
\hline ALRPH & 20 & 27 & 22 & 48 & 60 & 48 & 95 & 93 & 79 & 76 & 64 & 632 \\
\hline ANOBI & 5 & 2 & 2 & 1 & 2 & 0 & 2 & 2 & 2 & 2 & 0 & 20 \\
\hline BRASU & 100 & 112 & 117 & 148 & 153 & 150 & 168 & 157 & 163 & 154 & 139 & 1.561 \\
\hline CAB.SP & 0 & 0 & 0 & 0 & 0 & 1 & 0 & 0 & 4 & 0 & 2 & 7 \\
\hline COMDI & 0 & 3 & 5 & 4 & 2 & 4 & 10 & 9 & 5 & 0 & 6 & 48 \\
\hline CYPFE & 11 & 12 & 28 & 23 & 12 & 1 & 1 & 1 & 0 & 0 & 0 & 89 \\
\hline CYPGI & 4 & 12 & 13 & 19 & 19 & 12 & 17 & 11 & 6 & 6 & 9 & 128 \\
\hline CYPIR & 2 & 2 & 2 & 3 & 2 & 0 & 0 & 0 & 0 & 0 & 0 & 11 \\
\hline ECHGC & 0 & 4 & 3 & 2 & 0 & 2 & 0 & 0 & 0 & 0 & 0 & 11 \\
\hline ECHPO & 1 & 3 & 2 & 7 & 9 & 13 & 19 & 15 & 16 & 14 & 19 & 118 \\
\hline ECLAL & 0 & 5 & 11 & 19 & 2 & 2 & 1 & 1 & 0 & 0 & 1 & 42 \\
\hline EGEDE & 208 & 106 & 110 & 163 & 181 & 172 & 200 & 223 & 263 & 267 & 215 & 2.108 \\
\hline EICAZ & 133 & 136 & 145 & 173 & 161 & 165 & 171 & 187 & 177 & 190 & 188 & 1.826 \\
\hline EICCR & 4 & 7 & 13 & 30 & 45 & 23 & 21 & 20 & 19 & 21 & 30 & 233 \\
\hline ENISE & 0 & 0 & 0 & 0 & 0 & 2 & 3 & 0 & 0 & 2 & 3 & 10 \\
\hline FIMMI & 0 & 0 & 15 & 11 & 0 & 1 & 1 & 1 & 0 & 0 & 0 & 29 \\
\hline HEYCO & 2 & 4 & 4 & 3 & 3 & 2 & 5 & 4 & 2 & 2 & 2 & 33 \\
\hline HETRE & 2 & 0 & 4 & 0 & 0 & 0 & 0 & 0 & 0 & 0 & 0 & 6 \\
\hline HYVAM & 15 & 40 & 59 & 99 & 113 & 96 & 115 & 101 & 111 & 88 & 69 & 906 \\
\hline CLYAC & 13 & 10 & 9 & 12 & 13 & 10 & 16 & 14 & 16 & 15 & 12 & 140 \\
\hline LEMMI & 0 & 0 & 0 & 0 & 0 & 0 & 3 & 5 & 2 & 2 & 0 & 12 \\
\hline LIM.SP & 0 & 0 & 0 & 0 & 0 & 1 & 0 & 0 & 0 & 0 & 0 & 1 \\
\hline LUDOC & 3 & 6 & 5 & 6 & 3 & 2 & 5 & 3 & 3 & 4 & 4 & 44 \\
\hline LUDAC & 9 & 0 & 0 & 0 & 0 & 0 & 0 & 0 & 0 & 0 & 0 & 9 \\
\hline LUD.SP & 105 & 146 & 157 & 162 & 141 & 43 & 20 & 14 & 19 & 14 & 10 & 831 \\
\hline MYPBR & 27 & 27 & 24 & 26 & 36 & 37 & 74 & 63 & 45 & 48 & 29 & 436 \\
\hline OXYCU & 0 & 0 & 0 & 10 & 3 & 7 & 10 & 14 & 8 & 8 & 10 & 70 \\
\hline PANRV & 30 & 43 & 48 & 49 & 51 & 49 & 50 & 43 & 34 & 34 & 26 & 457 \\
\hline PASRP & 64 & 116 & 122 & 173 & 163 & 141 & 163 & 159 & 155 & 146 & 157 & 1.559 \\
\hline PIIST & 55 & 59 & 59 & 83 & 161 & 176 & 209 & 209 & 190 & 183 & 136 & 1.520 \\
\hline POLHP & 5 & 6 & 9 & 8 & 8 & 5 & 15 & 17 & 14 & 15 & 10 & 112 \\
\hline POLLA & 36 & 44 & 46 & 64 & 59 & 82 & 106 & 119 & 121 & 115 & 122 & 914 \\
\hline POFCL & 63 & 70 & 77 & 100 & 117 & 102 & 95 & 95 & 110 & 94 & 92 & 1.015 \\
\hline POFPA & 0 & 4 & 7 & 4 & 4 & 4 & 4 & 3 & 4 & 4 & 4 & 42 \\
\hline PTMPE & 0 & 0 & 0 & 0 & 0 & 0 & 0 & 1 & 4 & 0 & 4 & 9 \\
\hline RHCAU & 4 & 2 & 2 & 2 & 2 & 1 & 0 & 0 & 0 & 0 & 0 & 13 \\
\hline SAGMO & 182 & 171 & 164 & 187 & 181 & 147 & 95 & 74 & 82 & 90 & 94 & 1.467 \\
\hline SAVHE & 176 & 215 & 208 & 231 & 265 & 293 & 280 & 260 & 236 & 277 & 270 & 2.711 \\
\hline THAGE & 1 & 0 & 0 & 0 & 0 & 0 & 0 & 0 & 0 & 0 & 0 & 1 \\
\hline THY.SP & 1 & 0 & 0 & 0 & 3 & 3 & 2 & 2 & 4 & 2 & 2 & 19 \\
\hline
\end{tabular}


apresentaram fraca contribuição na caracterização do comportamento da comunidade de macrófitas aquáticas nesse periodo do ano. Entre as espécies com hábito flutuante, Eichhornia azurea apresentou maior importância na colonização do reservatório nos meses de agosto a dezembro do que as populações de Pistia stratiotes e Eichhornia crassipes. O aguapé-de-cordão, por ter um nicho ecológico semelhante ao das outras populações flutuantes, provavelmente apresentou maior potencial competitivo na colonização da lâmina d’água. Os meses de março, abril e julho foram caracterizados por populações de Hedychium coronarium, Panicum rivulare e Cyperus giganteus, apresentando características consideradas de transição entre os grupos de meses localizados nas extremidades do primeiro componente principal.

A rede neural de Kohonen dividiu as populações de macrófitas aquáticas que colonizaram o reservatório nos diferentes meses do ano em grupos de similaridade. Várias análises foram realizadas. A rede neural proporcionou várias possibilidades de agrupamento das espécies no tempo. Entre as várias classificações feitas pela rede neural, apresentadas nas Tabelas 3 a 5, foi escolhida aquela composta de nove grupos. Essa escolha foi baseada no conhecimento das espécies presentes nos grupos e nos levantamentos realizados pelo

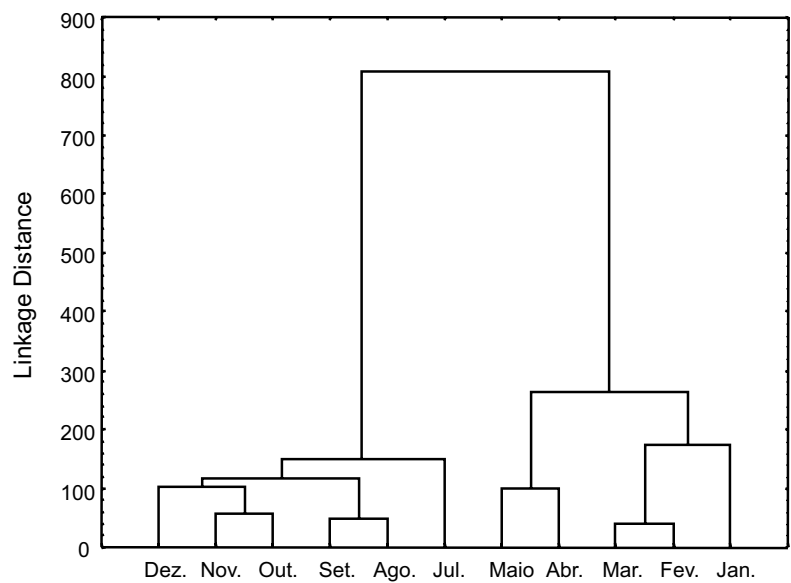

Figura 1 - Dendrograma mostrando a semelhança entre as comunidades macrófitas aquáticas que caracterizam cada um dos meses de 2004, no reservatório de Santana, Piraí-RJ. Quanto menores as barras que ligam dois meses, maior será a semelhança entre estes. pesquisador durante o ano de 2004. As tabelas apresentam os valores médios da frequência de cada grupo. Os valores presentes na frente de cada combinação população-período

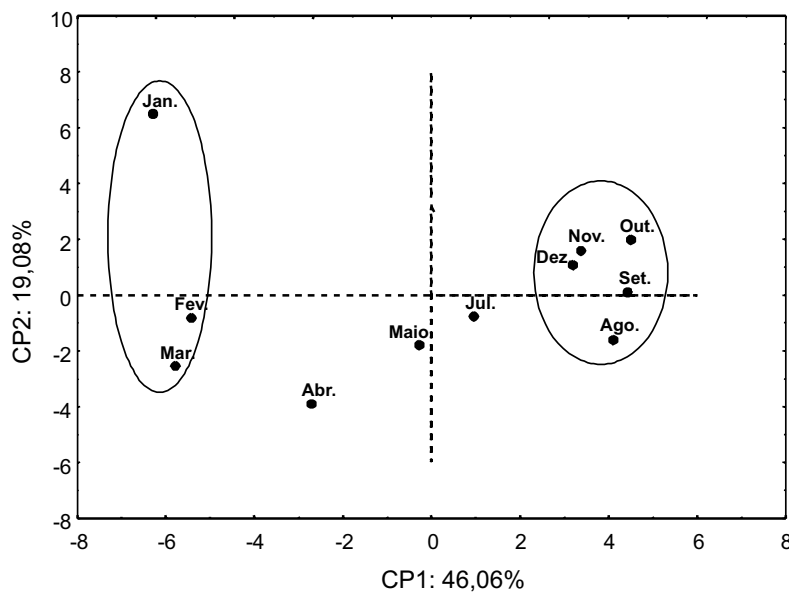

Figura 2 - Análise de componentes principais, demonstrando uma maior semelhança entre alguns meses com relação às populações de macrófitas aquáticas presentes no reservatório de Santana, Piraí-RJ, e suas respectivas densidades, no ano de 2004 .

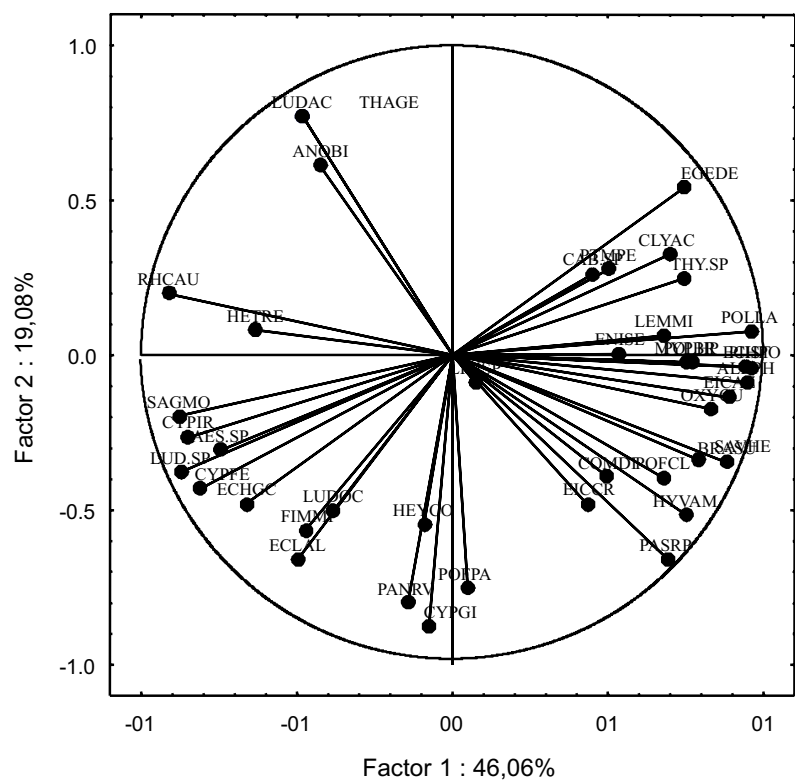

Figura 3 - Projeção das populações de macrófitas aquáticas que contribuem para a semelhança observada na comunidade de macrófitas aquáticas presentes nos diferentes meses do ano, apresentada pela análise de componentes principais. Quanto mais próxima da extremidade estiver uma população, maior será seu poder discriminatório. 
representam sua frequência dentro do referido grupo. Os valores presentes na frente do grupo representam sua frequência em relação à dos demais grupos. Segundo os resultados da rede de Kohonen, as populações de Egeria densa e Salvinia herzogii mostraram o mesmo comportamento quanto à colonização do reservatório nos meses do ano, com exceção do mês de janeiro para Salvinia herzogii e dos meses de fevereiro e março para Egeria densa, quando foram classificadas no grupo 2. Nestes meses a população de Egeria apresentou os mais baixos valores de colonização (Tabela 1). O mesmo comportamento pode ser atribuído à população de Salvinia herzogii, que no mês de janeiro apresentou a mais baixa nota de colonização (176) em comparação com os outros meses (acima de 200). No segundo grupo foram agrupadas as populações de Eichhornia crassipes, Paspalum repens e Pistia stratiotes. Esta última esteve presente neste grupo somente a partir de certo estádio da evolução da comunidade, cronologicamente marcado pelo mês de maio. Nos primeiros meses do ano, devido à sua baixa nota de colonização, esta espécie foi classificada no grupo 8 .

Tabela 3 - Populações de macrófitas aquáticas separadas em grupos de comportamento semelhante no reservatório de Santana, PiraíRJ, no ano de 2004, conforme análise realizada pelas Redes Neurais de Kohonen. Grupos 1, 2 e 3

\begin{tabular}{|c|c|c|c|c|c|}
\hline GRUPO 1 & $\begin{array}{c}233,0 \\
(233,0)\end{array}$ & GRUPO 2 & $\begin{array}{c}164,2 \\
(164,2)\end{array}$ & GRUPO 3 & $\begin{array}{c}158,5 \\
(158,5)\end{array}$ \\
\hline EGEDE_JAN & 208,0 & EICAZ_JAN & 133 & LUD.SP_JAN & 105 \\
\hline SAVHE_FEV & 215,0 & SAVHE_JAN & 176 & SAGMO_JAN & 182 \\
\hline SAVHE_MAR & 208,0 & EICAZ_FEV & 136 & LUD.SP_FEV & 146 \\
\hline EGEDE_ABR & 163,0 & PASRP_FEV & 116 & SAGMO_FEV & 171 \\
\hline SAVHE_ABR & 231,0 & EICAZ_MAR & 145 & LUD.SP_MAR & 157 \\
\hline EGEDE_MAI & 181,0 & PASRP_MAR & 122 & SAGMO_MAR & 164 \\
\hline SAVHE_MAI & 265,0 & EICAZ_ABR & 173 & LUD.SP_ABR & 162 \\
\hline EGEDE_JUL & 172,0 & PASRP_ABR & 173 & SAGMO_ABR & 187 \\
\hline SAVHE_JUL & 293,0 & EICAZ_MAI & 161 & LUD.SP_MAI & 141 \\
\hline EGEDE_AGO & 200,0 & PASRP_MAI & 163 & SAGMO_MAI & 181 \\
\hline SAVHE_AGO & 280,0 & PIIST_MAI & 161 & SAGMO_JUL & 147 \\
\hline EGEDE_SET & 223,0 & EICAZ_JUL & 165 & & \\
\hline SAVHE_SET & 260,0 & PASRP_JUL & 141 & & \\
\hline EGEDE_OUT & 263,0 & PIIST_JUL & 176 & & \\
\hline SAVHE_OUT & 236,0 & EICAZ_AGO & 171 & & \\
\hline EGEDE_NOV & 267,0 & PASRP_AGO & 163 & & \\
\hline SAVHE_NOV & 277,0 & PIIST_AGO & 209 & & \\
\hline EGEDE_DEZ & 215,0 & EICAZ_SET & 187 & & \\
\hline \multirow[t]{11}{*}{ SAVHE_DEZ } & 270,0 & PASRP_SET & 159 & & \\
\hline & & PIIST_SET & 209 & & \\
\hline & & EICAZ_OUT & 177 & & \\
\hline & & PASRP_OUT & 155 & & \\
\hline & & PIIST_OUT & 190 & & \\
\hline & & EICAZ_NOV & 190 & & \\
\hline & & PASRP_NOV & 146 & & \\
\hline & & PIIST_NOV & 183 & & \\
\hline & & EICAZ_DEZ & 188 & & \\
\hline & & PASRP_DEZ & 157 & & \\
\hline & & PIIST_DEZ & 136 & & \\
\hline
\end{tabular}


Tabela 4 - Populações de macrófitas aquáticas separadas em grupos de comportamento semelhante no reservatório de Santana, PiraíRJ, no ano de 2004, conforme análise realizada pelas Redes Neurais de Kohonen. Grupos 4, 5 e 6

\begin{tabular}{|c|c|c|c|c|c|}
\hline GRUPO 4 & 92,3 & GRUPO 5 & 141,9 & GRUPO 6 & 79,5 \\
\hline POFCL_JAN & 63 & BRASU_JAN & 100 & POLLA_JAN & 36 \\
\hline POFCL_FEV & 70 & BRASU_FEV & 112 & POLLA_FEV & 44 \\
\hline POFCL_MAR & 77 & BRASU_MAR & 117 & HYVAM_MAR & 59 \\
\hline POFCL_ABR & 100 & BRASU_ABR & 148 & POLLA_MAR & 46 \\
\hline POFCL_MAI & 117 & BRASU_MAI & 153 & POLLA_ABR & 64 \\
\hline POFCL_JUL & 102 & BRASU_JUL & 150 & POLLA_MAI & 59 \\
\hline POFCL_AGO & 95 & BRASU_AGO & 168 & POLLA_JUL & 82 \\
\hline POFCL_SET & 95 & BRASU_SET & 157 & POLLA_AGO & 106 \\
\hline POFCL_OUT & 110 & BRASU_OUT & 163 & POLLA_SET & 119 \\
\hline POFCL_NOV & 94 & BRASU_NOV & 154 & POLLA_OUT & 121 \\
\hline POFCL_DEZ & 92 & BRASU_DEZ & 139 & ALRPH_NOV & 76 \\
\hline & & & & POLLA_NOV & 115 \\
\hline & & & ALRPH_DEZ & 64 \\
\hline & & & POLLA_DEZ & 122 \\
\hline
\end{tabular}

O grupo 3 contém Ludwigia sp. e Sagittaria montevidensis nas densidades populacionais observadas até maio para a primeira e até julho para a segunda. O grupo 4 foi composto apenas por Pontederia lanceolata em todos os meses do ano; o grupo 5, apenas por Brachiaria arrecta; e o grupo 7, apenas por Panicum rivulare. O grupo 6 foi formado pela população de Polygonum lapathifolium. Espécies que não apresentaram padrões muito bem definidos de colonização foram inseridas nos grupos 8 e 9 , onde se situam importantes macrófitas aquáticas do histórico do reservatório, como Eichhornia crassipes.

Essa divisão dos grupos de colonização se deve a atributos característicos do comportamento invasor dessa espécie neste reservatório específico. Por exemplo, Salvinia herzogii e Egeria densa foram incluídas no mesmo grupo de colonização, apesar das diferenças no hábito de crescimento (uma é flutuante e a outra é submersa) e na morfologia. No entanto, essas espécies apresentam o mesmo ciclo de colonização no reservatório, ou seja, têm suas maiores densidades populacionais na mesma época. Espécies como Sagittaria montevidensis e Ludwigia sp, (especialmente Ludwigia repens) são colonizadoras primárias e substituídas por outras na evolução temporal da comunidade. Por isso, formaram um grupo conciso no primeiro semestre de avaliações. Para melhor visualização dos resultados, as frequências das populações que se destacaram nos primeiros três grupos de Kohonen foram utilizadas para o processamento de uma análise de componentes principais, juntamente com a projeção das variáveis representadas pelas espécies presentes nos grupos de Kohonen.

Os resultados apresentados na Figura 4 mostraram que os meses do ano podem ser agrupados em três grupos. No grupo localizado à esquerda do primeiro componente principal estão englobadas as espécies que mais caracterizam os meses de janeiro, fevereiro e março. Nesses três meses de amostragem se destacam as populações de Sagittaria montevidensis e Pontederia lanceolata, que colonizavam áreas marginais e rapidamente se estabeleceram no primeiro estádio seral. O comportamento dessas espécies nesses meses foi o fator de maior contribuição para a semelhança entre estes. Outras espécies, como Brachiaria arrecta, Panicum rivulare e Pontederia lanceolata, provavelmente apresentam plasticidade fenotípica suficiente para manter sua taxa de colonização, independentemente do estádio evolutivo da comunidade de macrófitas e do tipo de habitat disponibilizado para seu estabelecimento e crescimento. Esse comentário pode ser estendido a Polygonum lapathifolium. São, portanto, populações que podem ser encontradas como muito comuns nos vários estádios 
Tabela 5 - Populações de macrófitas aquáticas separadas em grupos de comportamento semelhante no reservatório de Santana, Piraí-RJ, no ano de 2004, conforme análise realizada pelas Redes Neurais de Kohonen. Grupos 7, 8 e 9

\begin{tabular}{|c|c|c|c|c|c|}
\hline GRUPO 7 & 41,5 & GRUPO 8 & 75,8 & GRUPO 9 & 5,0 \\
\hline PANRI_JAN & 30 & PASRP_JAN & 64 & AESDE._JAN & 1 \\
\hline PANRI_FEV & 43 & PIIST_JAN & 55 & ALRPH_JAN & 20 \\
\hline PANRI_MAR & 48 & EGEDE_FEV & 106 & ANOBI_JAN & 5 \\
\hline PANRI_ABR & 49 & PIIST_FEV & 59 & CAB.SP_JAN & 0 \\
\hline PANRI_MAI & 51 & EGEDE_MAR & 110 & COMDI_JAN & 0 \\
\hline PANRI_JUL & 49 & PIIST_MAR & 59 & CYPFE_JAN & 11 \\
\hline PANRI_AGO & 50 & ALRPH_ABR & 48 & CYPGI_JAN & 4 \\
\hline PANRI_SET & 43 & HYVAM_ABR & 99 & CYPIR_JAN & 2 \\
\hline PANRI_OUT & 34 & PIIST_ABR & 83 & ECHGC_JAN & 0 \\
\hline PANRI_NOV & 34 & ALRPH_MAI & 60 & ECHPO_JAN & 1 \\
\hline \multirow[t]{25}{*}{ PANRI_DEZ } & 26 & EICCR_MAI & 45 & ECLAL_JAN & 0 \\
\hline & & HYVAM_MAI & 113 & EICCR_JAN & 4 \\
\hline & & MYPBR_MAI & 36 & ENISE_JAN & 0 \\
\hline & & ALRPH_JUL & 48 & FIMMI_JAN & 0 \\
\hline & & HYVAM_JUL & 96 & HEYCO_JAN & 2 \\
\hline & & LUD.SP_JUL & 43 & HETRE_JAN & 2 \\
\hline & & MYPBR_JUL & 37 & HYVAM_JAN & 15 \\
\hline & & ALRPH_AGO & 95 & CLYAC_JAN & 13 \\
\hline & & HYVAM_AGO & 115 & LEMMI_JAN & 0 \\
\hline & & MYPBR_AGO & 74 & LIM.SP_JAN & 0 \\
\hline & & SAGMO_AGO & 95 & LUDOC_JAN & 3 \\
\hline & & ALRPH_SET & 93 & LUDAC_JAN & 9 \\
\hline & & HYVAM_SET & 101 & MYPBR_JAN & 27 \\
\hline & & MYPBR_SET & 63 & OXYCU_JAN & 0 \\
\hline & & SAGMO_SET & 74 & POLHP_JAN & 5 \\
\hline & & ALRPH_OUT & 79 & POFPA_JAN & 0 \\
\hline & & HYVAM_OUT & 111 & PTMPE_JAN & 0 \\
\hline & & MYPBR_OUT & 45 & RHCAU_JAN & 4 \\
\hline & & SAGMO_OUT & 82 & THAGE_JAN & 1 \\
\hline & & HYVAM_NOV & 88 & THY.SP_JAN & 1 \\
\hline & & MYPBR_NOV & 48 & AESDE_FEV & 2 \\
\hline & & SAGMO_NOV & 90 & ALRPH_FEV & 27 \\
\hline & & HYVAM_DEZ & 69 & ANOBI_FEV & 2 \\
\hline & & SAGMO_DEZ & 94 & CAB.SP_FEV & 0 \\
\hline & & & & Etc... & \\
\hline
\end{tabular}

serais da comunidade de macrófitas. Num segundo grupo, localizado à direita do primeiro componente principal, foram agregadas as populações que mais caracterizaram os meses de agosto, setembro, outubro, novembro e dezembro. A este grupo foram associadas inúmeras espécies, que são um reflexo da heterogeneidade espacial que se estabeleceu com a colonização do reservatório de Santana.
As espécies associadas a esta época de amostragem foram Egeria densa, Paspalum repens, Eichhornia azurea, Salvinia herzogii, Brachiaria arrecta e Polygonum lapathifolium. Em um terceiro grupo estão segregadas as espécies que mais caracterizaram os meses de maio, abril e julho e há a associação das populações de Pistia stratiotes, Ludwigia sp. e, também, Sagittaria montevidensis e Pontederia 


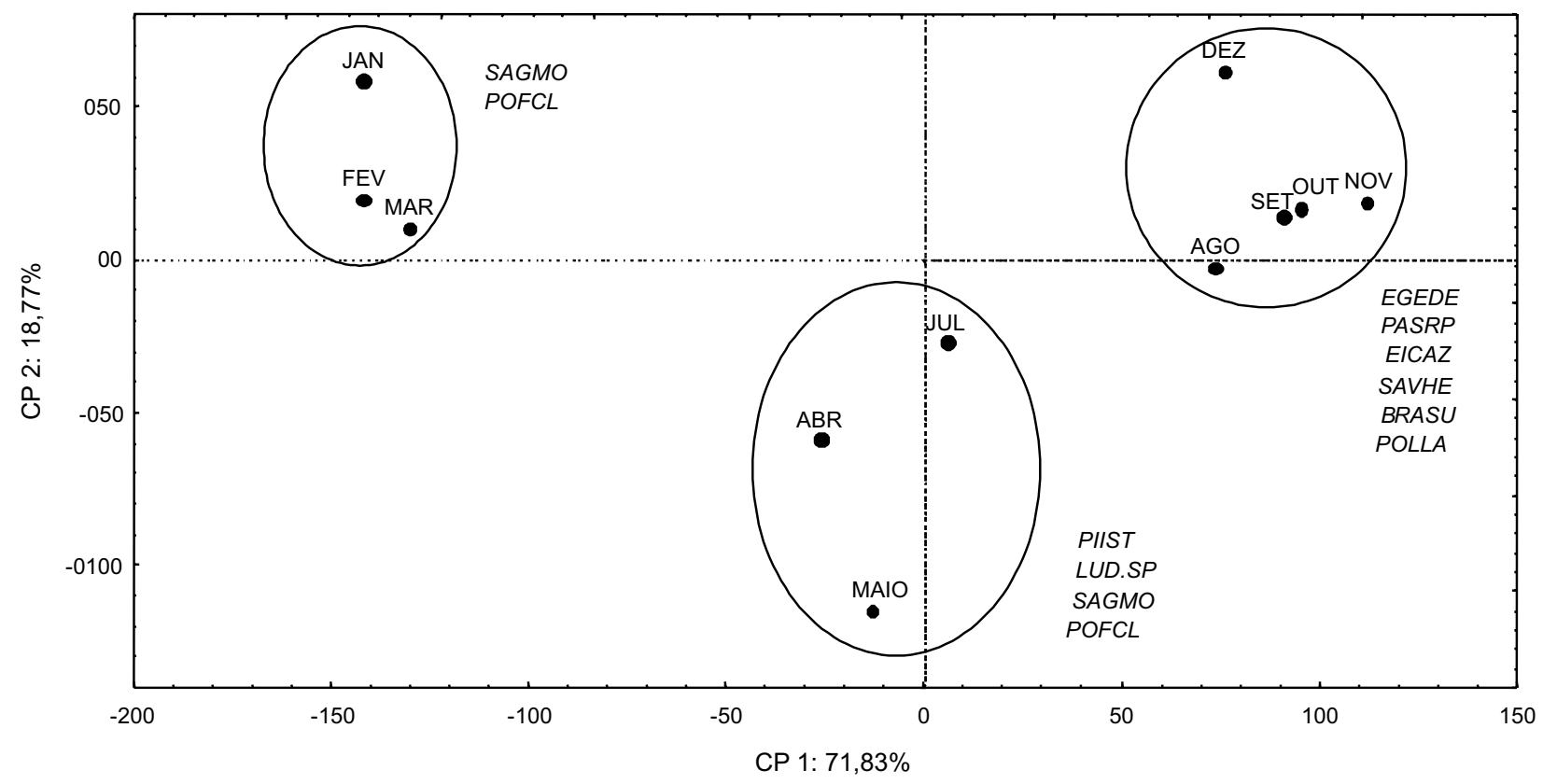

Figura 4 - Análise de componentes principais, utilizando os dados de frequência das espécies presentes nos grupos 1, 2 e 3 de Kohonen, demonstrando uma maior semelhança entre alguns meses com relação às populações de macrófitas aquáticas presentes no reservatório de Santana, Piraí-RJ, e suas respectivas densidades, no ano de 2004.

lanceolata. Estas duas espécies já foram importantes no primeiro grupo e, outra vez, evidenciam a possibilidade de espécies com elevada plasticidade fenotípica para colonização de diferentes estádios serais da sucessão.

As várias abordagens de análise dos resultados do levantamento das populações de macrófitas aquáticas no ano de 2004 demonstraram que houve uma bem definida sucessão de condições de colonização no reservatório, que permite a definição de estádios serais para fins de elaboração de planos de manejo.

\section{LITERATURA CITADA}

COSTA, N. V.; RODELLA, R. A.; MARTINS, D.

Diferenciação de espécies daninhas aquáticas pela análise multivariada de caracteres estruturais foliares. Planta Daninha, v. 24, n. 1, p. 13-20, 2006

CHON, T. S. et al. Patternizing communities by using an artificial neural network. Ecol. Model., v. 90, n. 1, p. 69-78, 1996.

COLON-GAUD, J. C.; KELSO, W. E.; RUTHERFORD, D A. Spatial distribution of macroinvertebrates inhabiting hydrilla and coontail beds in the Atchafalaya Basin, Louisiana J. Aquat. Plant Manag., v. 42, n. 1, p. 85-91, 2004.
HAIR, J. F. et al. Análise multivariada de dados. 5.ed. Porto Alegre: 2005.

HUNTINGFORD, C.; COX, P. M. Use of statistical and neural networks techniques to detect how stomatal conductance responds to changes in the local environment Ecol. Model., v. 97, n. 3, p. 217-246, 1996.

KOHONEN, T. Self-organization and associative memory. 3.ed. New York: Springer-Verlag, 1989. 311 p.

KOHONEN, T. The self-organizing map. Proceedings IEEE, v. 78, n. 9, p. 1464-1480, 1990.

LEK, S. et al. Application of neural networks to modelling non-linear relationships in ecology. Ecol. Model., v. 90, n. 1, p. 39-52, 1996.

LEVINE, E. R.; KIMES, D. S.; SIGLLITO, V. G. Classifying soil structure using neural networks. Ecol. Model., v. 92, n. 1, p. 101-108, 1996.

POMPÊO, M. L. M. et al. Heterogeneidade espacial do fitoplâncton no reservatório de Boa Esperança (MaranhãoPiauí, Brasil). Acta Limnol. Bras., v. 10, n. 2, p. 101-113, 1998.

WARD, J. H. Hierarchical grouping to optimize an objective function. J. Am. Statist. Assoc., v. 58, n. 301, p. $236,1963$. 